'Departamento de Medicina. Escuela de Medicina de la Facultad de Medicina de la Pontificia Universidad Católica de Chile.

${ }^{2}$ Departamento de Dermatología. Escuela de Medicina, Facultad de Medicina de la Pontificia Universidad Católica de Chile. ${ }^{3}$ Departamento de Anatomía Patológica. Escuela de Medicina de la Facultad de Medicina de la Pontificia Universidad Católica de Chile. anterna de Medicina. Escuela de Medicina, Facultad de Medicina de la Pontificia Universidad Católica de Chile.

Recibido el 4 de mayo de 2015 , aceptado el 11 de noviembre de 2015.

Correspondencia a:

Dr. Gonzalo Eymin Lago Hospital Clínico Red de Salud UC-Christus Marcoleta $367,5^{\circ}$ piso, Santiago, Chile. Teléfono: +562 23543152

\section{Eritema nodoso: Causas más prevalentes en pacientes que se hospitalizan para estudio, $y$ recomendaciones para el diagnóstico}

\author{
PABLO VARAS ${ }^{1}$, ANDREA ANTÚNEZ-LAY ${ }^{\text {a }}$, JOSÉ MIGUEL BERNUCCI ${ }^{1}$, \\ LAURA COSSIO ${ }^{2}$, SERGIO GONZÁLEZ ${ }^{3}$, GONZALO EYMIN ${ }^{1}$

\section{Erythema nodosum. Analysis of 91 hospitalized patients}

Background: Erythema Nodosum (EN) is an acute nodular erythematous cutaneous eruption. It is presumed to be a hypersensitivity reaction and it may be idiopathic, or occur in association with diverse diseases and medications. Aim: To identify the main etiologies of EN among patients admitted to a medicine service of a clinical hospital. Material and Methods: Analysis of medical records of patients discharged with the diagnosis of $\mathrm{EN}$ during a ten years period. $\mathrm{Pa}$ tients were considered to have secondary EN when an underlying condition was found, and to have idiopathic EN when no such condition was found. Results: One hundred twenty nine patients were identified, but 91, aged $38 \pm 14$ years ( $86 \%$ females) had available clinical information. The etiology was idiopathic in $32 \%$ and secondary in $68 \%$ of patients. The leading etiologies were post-Streptococcal in $32 \%$, followed by sarcoidosis in $11 \%$, primary tuberculosis in 7\%, Mycoplasma infection in 3\%, Bartonella infection in 3\%, Behçet's syndrome in $2 \%$, inflammatory bowel disease in $2 \%$, drugs in $1 \%$ and other etiologies in $8 \%$. Secondary cases of EN had higher levels of C Reactive Protein and leucocytes than idiopathic cases. Conclusions: The distribution of causes in our study population were similar to those reported in the literature (With smaller proportion of idiopathic), as was the man/woman (1/6) ratio, and the preponderance of cases in winter and spring. Infectious diseases were the leading causes of EN in this group of patients, and a reasonable initial approach in the hospital should include a diligent medical history and physical examination, complete blood count, ESR, titles ASO, PPD tuberculin skin test, chest X-ray or CT scan and, where deemed appropriate, specific autoimmune and infectious serology.

(Rev Med Chile 2016; 144: 162-168)

Key words: Erythema nodosum; Inflammatory bowel diseases; Sarcoidosis; Streptococcal Infections; Tuberculosis.
$\mathrm{E}$ 1 eritema nodoso (EN) es el tipo de paniculitis más frecuente. Se presenta como nódulos eritematosos violáceos de 1 a 5 $\mathrm{cm}$, profundos, dolorosos, preferentemente en la cara anterior de las piernas, que evolucionan con apariencia de equimosis (coloración inicialmente violácea, luego amarillo-verdosa) sin dejar cicatriz, desapareciendo en un período de 2 a 8 semanas. Las lesiones también pueden aparecer en muslos, tronco y extremidades superiores, pero su ausencia en piernas es poco habitual (Figura 1). Además de dolor local es frecuente la asociación de fiebre, 
compromiso del estado general y poliartralgias. El principal diagnóstico diferencial lo constituye el eritema indurado de Bazin, que suele comprometer la parte posterior de las piernas y tiende a ulcerarse más. Otros diagnósticos diferenciales son la tromboflebitis superficial, las celulitis y las vascultis cutáneas. En el EN suele haber elevación de la velocidad de eritrosedimentación en hasta $80 \%$ de los casos. En la histopatología se observa una paniculitis septal, sin compromiso del centro del lobulillo, con un infiltrado de predominio linfocítico, con algunas células gigantes, eosinófilos e histiocitos, además de neutrófilos en las formas precoces. No hay vasculitis asociada, pero sí puede haber cambios inflamatorios inespecíficos en vasos pequeños $^{1-3}$. Su incidencia varía entre 1 y 5 por 100.000 habitantes y la distribución por género es predominante en mujeres, con una relación mujeres es a hombres de 3 a 6 es a 1, según las distintas series ${ }^{4-6}$. Afecta principalmente al grupo etario entre los 10 y 50 años, con un pico entre los 20 y 40 años, sin embargo puede presentarse a cualquier edad ${ }^{1,2,7}$. La patogenia exacta es incierta, si bien se sabe que existiría a nivel hipodérmico una reacción de hipersensibilidad retardada (tipo IV) secundaria a antígenos de diferente origen, se han descrito además depósito de complejos inmunes y anticuerpos en ciertas etiologías. Sin embargo, a la fecha no se tiene claro el rol de cada uno de estos mecanismos en el desarrollo y recurrencia del EN. La identificación del agente causal en EN puede resultar particularmente difícil, encontrándose asociación con cuadros de origen infeccioso, inflamatorio, autoinmune, asociada al uso de fármacos (principalmente anticonceptivos, amoxicilina y sulfonamidas), terapias hormonales y el embarazo. A pesar de esto, muchas veces no se logra establecer la causa y no son pocos los

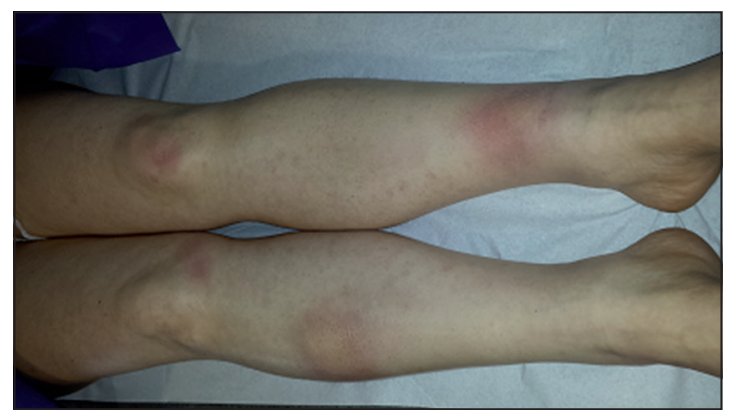

Figura 1. Eritema nodoso. casos de origen idiopático ${ }^{4,8,9}$. Adicionalmente, la mayoría de los estudios muestran que la etiología varía dependiendo de la época en que se llevan a cabo, de la epidemiología, y de la carga de enfermedad en las distintas áreas geográficas estudiadas, no existiendo a la fecha guías de práctica clínica que orienten en el estudio etiológico. Por lo anterior, nos parece muy importante conocer la distribución de causas encontradas en nuestro medio en pacientes con EN, de modo de generar recomendaciones para hacer un estudio costo efectivo basado en la mejor evidencia disponible.

\section{Materiales y Métodos}

Estudio retrospectivo de pacientes mayores de 15 años con diagnóstico de eritema nodoso atendidos entre los años 2002 y 2012 en el Servicio de Medicina Interna del Hospital Clínico de la Pontificia Universidad Católica de Chile. Los pacientes se obtuvieron a partir de los registros de alta hospitalaria de nuestra institución y registro de biopsias del Departamento de Anatomía Patológica del Hospital Clínico. Se contó con aprobación del comité de ética institucional y se autorizó la dispensa de consentimiento informado, dada la naturaleza observacional del estudio y a que los identificadores fueron destruidos una vez extraídos los datos (Se solicitó consentimiento a paciente fotografiado). Se revisaron fichas clínicas, sistemas de exámenes de sangre y de radiología (en papel o electrónica). Se obtuvieron datos demográficos, características clínicas, exámenes de laboratorio, imágenes, resultados de biopsias (si disponible) y el tratamiento inicial indicado. El diagnostico de EN se estableció por criterios clínicos, o por estudio histológico compatible cuando estuvo disponible. Las etiologías se definieron según los siguientes criterios:

Post estreptocócica (PS): Cuadro clínico de faringoamigdalitis o infección de partes blandas dentro de las 6 semanas previas al cuadro de EN más títulos de anti estreptolisina $\mathrm{O}(\mathrm{ASO})$ elevado; adicionalmente se definió como cuadro probable al cuadro clínico descrito con ASO (-) en contexto de uso previo de antibióticos, o bien, a títulos de ASO elevados sin clínica de infección estreptocócica reportada ${ }^{10}$.

Tuberculosis (TBC): A la presencia de prueba de tuberculina $(\mathrm{PPD})>10 \mathrm{~mm}$ o presencia de ba- 
ciloscopia o cultivo de Koch o determinación por técnica de biología molecular positiva. Se determinó TBC activa o TBC latente según evidencias de infección clínicamente activa.

Otras infecciones: Fueron definidas según la presencia de marcadores serológicos o cultivo de microorganismo, entre ellas búsqueda de $\mathrm{Myco-}$ plasma pneumoniae, Bartonella henselae, virus de Ebstein Barr, citomegalovirus, virus de inmunodeficiencia humana, virus hepatitis B y C.

Sarcoidosis: Se definió según la presencia de cuadro clínico-radiológico con biopsia de tejido compatible.

Enfermedad de Behçet: Cuadro clínico según los criterios diagnósticos del International Study Group for Behçet Disease ${ }^{11}$.

Enfermedad inflamatoria intestinal: Cuadro clínico endoscópico compatible y biopsia confirmatoria.

Otras enfermedades autoinmunes: según criterios clínicos y serológicos.

Drogas: Inicio dentro de 3 meses previo al cuadro de EN. Entre ellas: antibióticos (énfasis en penicilinas y sulfas), antinflamatorios no esteroidales, anticonceptivos orales, terapia de reemplazo hormonal, paracetamol, inhibidores de bomba de protones, entre otros.

Como causa idiopática se definió todos aquellos casos con estudio etiológico negativo, considerando como estudio mínimo: historia y examen físico en búsqueda de causas conocidas de EN, fármacos, estudio de TBC, ASO e imagen de tórax (radiografía o tomografía computarizada).

Se obtuvo el estudio de laboratorio incluyendo hemograma, velocidad de eritrosedimentación (VHS), proteína C reactiva (PCR), serologías autoinmunes y virales nivel de ASO y PPD, estudio de imágenes como radiografía de tórax y tomografía computarizada de estar disponibles. Adicionalmente se registró el tratamiento utilizado en el cuadro agudo.

Este estudio contó con la aprobación del Comité de Ética del Centro de Investigaciones Médicas de la Facultad de Medicina de nuestra Universidad.

\section{Análisis estadístico}

Las variables categóricas se expresaron en porcentajes y variables continuas a través de medias y desviación estándar (DE), en los casos de $n$ pequeño se expresó la mediana en vez de la media como debidamente se señala. Se realizó un análisis de variables continuas con test no paramétrico ante la duda de distribución normal de nuestra población utilizando el método de Mann Whitney, a fin de observar diferencias entre casos idiopáticos y secundarios de EN en relación a parámetros inflamatorios. En este caso un valor $\mathrm{p}<0,05$ se consideró estadísticamente significativo.

\section{Resultados}

Se encontraron 129 pacientes con diagnóstico inicial de EN, excluyéndose 34 casos por no encontrarse registros clínicos o por no tener el estudio mínimo previamente descrito, y 4 pacientes por diagnóstico definitivo distinto a EN según biopsia cutánea, resultando la cohorte final en 91 pacientes. De estos, $84,6 \%$ eran mujeres (relación mujer:hombre $=6: 1$ ), con una media de edad $38 \pm 14$ años (rango 16-76 años). El 67\% de los pacientes se presentó con fiebre $\left(>38^{\circ} \mathrm{C}\right)$, $74 \%$ con artralgias y $21 \%$ con tos. A 50 pacientes se les practicó biopsia cutánea según criterio del tratante. El compromiso cutáneo fue el motivo de consulta inicial en $82 \%$ de los casos, siendo en todos ellos lesiones bilaterales en extremidades inferiores (sólo 4 casos presentaron además compromiso de extremidades superiores). En cuanto a la etiología, $68,1 \%$ de los casos se consideró secundario y $31,9 \%$ idiopático. El 48,35\% de los casos se consideró asociado a infección: 31,9\% post estreptocócico, 6,6\% tuberculosis (4 pacientes TBC latente, 2 TBC activa ganglionar) y 18,7\% a enfermedades autoinmunes (11\% sarcoidosis). Sólo 1 caso se estimó que fue secundario a drogas (Tabla 1). Dentro del grupo post estreptocócico (29 casos) se identificaron 8 casos $(28 \%)$ como cuadro probable ( 3 con ASO elevado sin clínica y 5 con cuadro clínico con ASO negativo y uso de antibióticos previos), y los otros 21 (72\%) como definitivo. No se encontraron en nuestra serie casos de infección por virus de hepatitis $\mathrm{B}$, C ni VIH, linfoma de Hodgkin, histoplasmosis, coccidiodomicosis, blastomicosis ni yersiniosis.

De los 27 casos con ASO (+), destacan dos casos de sarcoidosis confirmada y un caso de infección por Mycoplasma pneumoniae.

Las características de la población estudiada, su distribución etiológica (secundario vs idiopático) y estacionalidad de aparición se detallan en 
la Tabla 1 y Figura 2 respectivamente. Dentro del análisis de causa se establecieron diferencias en los valores de parámetros inflamatorios (leucocitosis, VHS y PCR) (Tabla 2), destacando que los casos secundarios tuvieron valores de VHS y leucocitosis significativamente más altos que los casos idiopáticos $(\mathrm{p}<0,05)$.

El estudio de laboratorio para etiologías infecciosas y autoinmunes se detalla en las Tablas $3 \mathrm{~A} \mathrm{y}$ $3 \mathrm{~B}$, respectivamente.

Con respecto al tratamiento, los casos de causa secundaria recibieron tratamiento según la etiología identificada, los de origen infeccioso fueron tratados con antibióticos, incluyendo inicio de tratamiento anti-TBC o quimioprofilaxis en los 6 casos correspondientes. En 7 pacientes de causa idiopática se dio tratamiento antibiótico empírico. El manejo farmacológico consistió principalmente en analgesia con antiinflamatorios no esteroidales $(56,9 \%)$, paracetamol $(39,5 \%)$, yoduro de potasio $(11,6 \%)$, opiáceos $(4,3 \%)$, corticoides orales $(33,7 \%)$, colchicina $(4,3 \%)$. Un paciente recibió pentoxifilina y otro azatriopina.

En nuestros registros no fue posible establecer con certeza el porcentaje de recurrencia de EN ni el tiempo de resolución del cuadro.

Tabla 1. Características demográficas y causas de EN separados entre causas idiopáticas y secundarias

\begin{tabular}{|lcccccc|}
\hline Etiologías EN & $\mathbf{n}$ & $\mathbf{\%}$ & Edad & Rango etario & Sexo (M/F) & Biopsia (n) \\
\hline Idiopático & 29 & 31,87 & 41,34 & $(17-76)$ & $(3 / 26)$ & 18 \\
Secundario & 62 & 68,13 & 37,32 & $(16-72)$ & $(11 / 51)$ & 32 \\
1. Infeccioso & 44 & 48,35 & 37,9 & $(16-72)$ & $(8 / 36)$ & 27 \\
PS & 29 & 31,86 & 35,2 & $(16-60)$ & $(5 / 24)$ & 18 \\
TBC & 6 & 6,6 & 52,67 & $(34-72)$ & $(1 / 5)$ & 5 \\
Mycoplasma & 3 & 3,3 & 27,33 & $(18-33)$ & $(1 / 2)$ & 2 \\
Bartonella & 3 & 3,3 & 40 & $(28-50)$ & $(0 / 3)$ & 1 \\
Otros* & 3 & 3,3 & 424 & $(39-49)$ & $(1 / 2)$ & 1 \\
2. Autoinmune & 17 & 18,7 & 35,82 & $(22-61)$ & $(3 / 14)$ & 5 \\
Sarcoidosis & 10 & 11,0 & 36,2 & $(26-51)$ & $(3 / 7)$ & 4 \\
EII** & 2 & 2,2 & 28,5 & $(22-35)$ & $(0 / 2)$ & 0 \\
Enfermedad de Behçet & 2 & 2,2 & 29 & $(24-34)$ & $(0 / 2)$ & 0 \\
Otros*** & 3 & 3,3 & 44 & $(28-61)$ & $(0 / 3)$ & 1 \\
3. Drogas & 1 & 1,1 & 37 & & $(0 / 1)$ & 0 \\
\hline Total & 91 & & $38 \pm 14$ & $(16-76)$ & $(1: 6)$ & $(50)$ \\
\hline
\end{tabular}

*Otros infecciosos: uretritis por Streptococcus grupo B (SGB), espondilodiscitis sin agente identificado y Salmonellosis. ${ }^{* *}$ Enfermedad Inflamatoria Intestinal. ${ }^{* *}$ Otros Autoinmune: Enfermedad mixta del tejido conectivo, artritis reumatoídea juvenil, sobreposición hepatitis autoinmune y cirrosis biliar primaria.

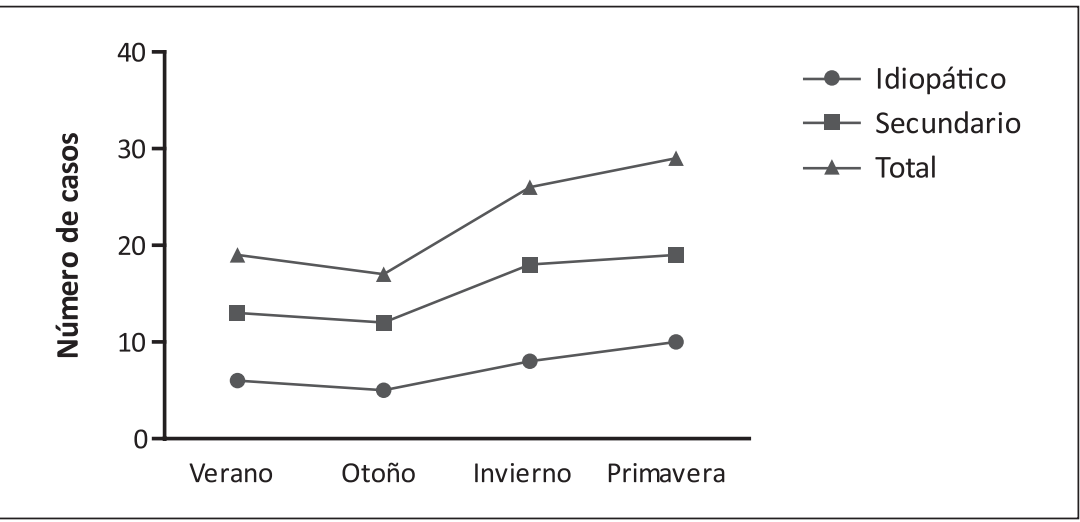

Figura 2. Estacionalidad. 
Tabla 2. Parámetros inflamatorios según causas de EN

\begin{tabular}{|lccc|}
\hline Etiologías EN & Leucocitos (media \pm DE) & VHS (media \pm DE) & PCR (media \pm DE) \\
Idiopático & $8.017 \pm 2.217(\mathrm{n}: 24)^{*}$ & $40,65 \pm 28,13(\mathrm{n}: 23)^{*}$ & $8,5 \pm 5,78(\mathrm{n}: 16)$ \\
Secundario & $9.842 \pm 3.537(\mathrm{n}: 55)^{*}$ & $59,14 \pm 34,11(\mathrm{n}: 57)^{*}$ & $9,26 \pm 6,98(\mathrm{n}: 45)$ \\
\hline 1. Infeccioso & $9.842 \pm 3.822(\mathrm{n}: 38)$ & $65,98 \pm 35,69(\mathrm{n}: 41)$ & $8,75 \pm 7,5(\mathrm{n}: 32)$ \\
PS & $10.052 \pm 3.315$ & $62,5 \pm 30,6$ & $9,04 \pm 5,28$ \\
TBC & $9.975 \pm 8.135$ & $62,8 \pm 32,9$ & $9,17 \pm 13,12$ \\
Mycoplasma** & 8.100 & 83 & 7,7 \\
Bartonella** & 10.200 & 76 & 4,3 \\
Otros & $\mathrm{NA}$ & $\mathrm{NA}$ & $\mathrm{NA}$ \\
2. Autoinmune & $9.806 \pm 2.993(\mathrm{n}: 16)$ & $39,46 \pm 21,62(\mathrm{n}: 15)$ & $10,13 \pm 7,27(\mathrm{n}: 12)$ \\
Sarcoidosis & $10.400 \pm 2872$ & $42,3 \pm 19,3$ & $10,59 \pm 5,64$ \\
Ell*** & 12,800 & 44 & 18,8 \\
Behçet*** & 13.300 & 30 & 3,75 \\
Otros & $\mathrm{NA}$ & $\mathrm{NA}$ & $\mathrm{NA}$ \\
3. Drogas & 10.400 & 74 & 15 \\
Total & $9.287 \pm 3.289$ & $53,83 \pm 33,41$ & $9,06 \pm 6,65$ \\
\hline
\end{tabular}

${ }^{*} \mathrm{p}<0,05 .{ }^{* *}$ : Se muestra la mediana dado $\mathrm{n}$ pequeño. ${ }^{* *}$ Se muestra la media, $\mathrm{n}=2$ no permite cálculo de mediana ni análisis de DE. NA: no aplica por múltiples patologías.

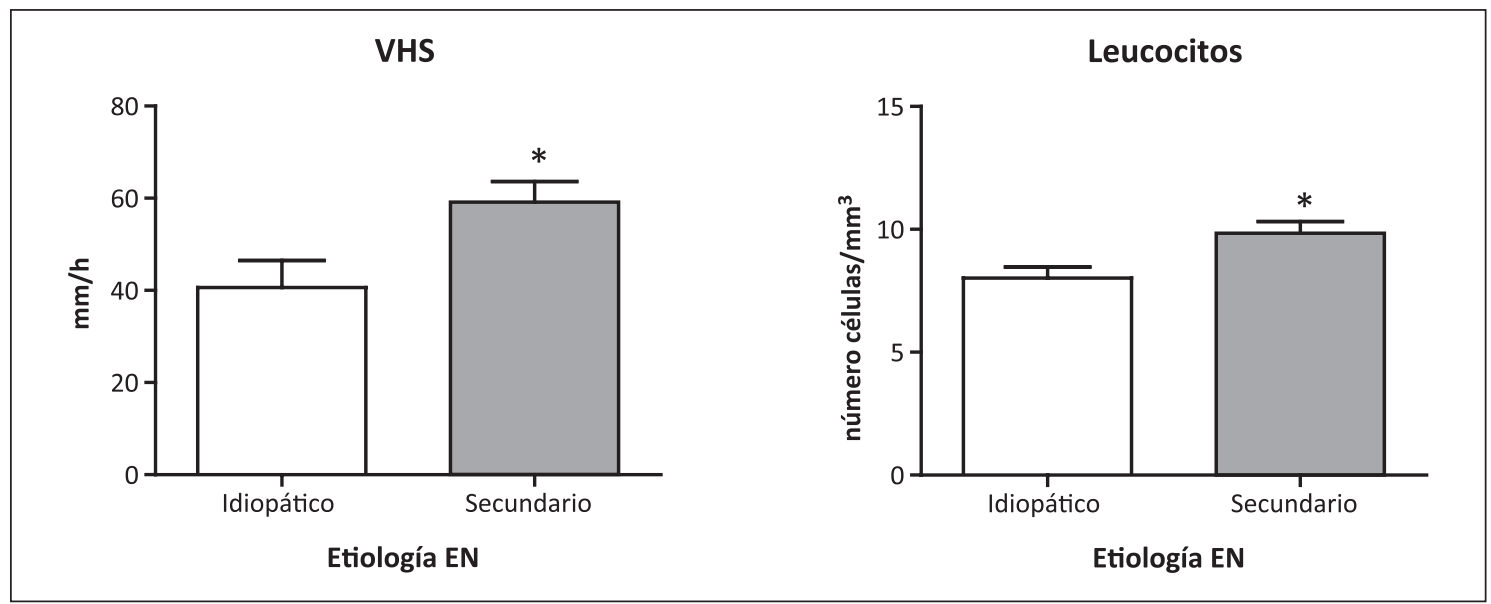

Figura 3. Parámetros inflamatorios. Diferencias entre etiologías idiopática y secundaria de EN. Se expresan medias y error estándar de la media. * $\mathrm{p}<0,05$.

\section{Discusión}

Las características demográficas de nuestra cohorte son similares a las reportadas en la mayoría de los estudios, con media de edad de 38 años y un rango amplio (16 a 75 años), siendo preferentemente una patología de predominio en mujeres (relación 6:1) y de mayor ocurrencia en invierno y primavera, tal cual se reporta en múltiples estudios. Dentro de las etiologías en- contradas, existe una menor proporción de causas idiopáticas a las reportadas en otros trabajos, sin embargo es posible que este dato tenga el sesgo de que sólo se incluyeron pacientes hospitalizados, en los que el estudio etiológico probablemente fue más exhaustivo que en pacientes que se estudian y manejan de manera ambulatoria. En relación al resto de causas, nuestra serie presenta de igual manera una alta proporción de causas infecciosas, con los cuadros post estreptocócicos como los 
Tabla 3A. Estudio etiológico infeccioso

\begin{tabular}{|lcc|}
\hline Estudio & $\begin{array}{c}\text { Solicitado } \\
\text { (n pacientes) }\end{array}$ & Positivo \\
\hline ASO & 59 & 27 \\
\hline VIH & 31 & 0 \\
\hline EBV & 12 & 0 \\
\hline CMV & 12 & 0 \\
\hline VHC & 21 & 0 \\
\hline VHB & 21 & 0 \\
\hline Mycoplasma & 10 & 3 \\
\hline Bartonella & 11 & 3 \\
\hline
\end{tabular}

más frecuentes, seguidos de TBC y una no menor proporción de cuadros secundarios a Mycoplasma o Bartonella, agentes previamente descritos pero de muy baja frecuencia. En nuestra serie los 2 casos potencialmente estreptocócicos (un caso de uretritis por Streptococcus grupo B (SGB) y un caso de espondilodiscitis sin agente identificado) no fueron considerados dentro de los post estreptocócicos clásicos, ya que estos hacen referencia principalmente a los cuadros secundarios a faringoamigdalitis purulenta por Streptococcus Beta hemolítico grupo A o a infección de partes blandas seguidas de positivización del ASO (ASO se eleva en grupo A y algunos $\mathrm{C}$ y $\mathrm{G}$ ) y no a infecciones mediadas por Streptococcus grupo $\mathrm{B}^{12}$.

En el grupo de causas autoinmunes, la sarcoidosis, al igual que en otros reportes, ocupa un lugar preponderante, seguida por la enfermedad de Behçet y cuadros de enfermedad inflamatoria intestinal, destacando en nuestros registro la aparición de un cuadro de enfermedad mixta del tejido conectivo, otro de artritis reumatoídea juvenil y un caso de síndrome de sobreposición hepatitis autoinmune y cirrosis biliar primaria, asociaciones descritas en la literatura ${ }^{13-15}$.

Respecto al laboratorio es interesante destacar que los parámetros inflamatorios, como leucocitosis y VHS, están significativamente más elevados en los casos secundarios que en los idiopáticos, sin encontrar diferencias significativas de éstos entre las distintas causas secundarias. No se detectaron diferencias significativas en los niveles de PCR.

Considerando los resultados obtenidos en el presente estudio, las causas infecciosas siguen
Tabla 3B. Estudio etiológico autoinmune

\begin{tabular}{|lcc|}
\hline Estudio & Solicitado & Positivo \\
\hline Anticuerpos antinucleares & 60 & 10 \\
\hline Niveles de C3 & 40 & 25 \\
\hline Niveles de C4 & 40 & 5 \\
\hline Factor reumatoideo & 31 & 1 \\
\hline Anti DNA & 30 & 1 \\
Perfil ENA & 21 & 0 \\
\hline ANCA & 35 & 0 \\
\hline
\end{tabular}

Niveles de C3 y C4 son niveles elevados y en múltiples condiciones. ANA: 3 idiopáticos, 2 TBC, 2 Sarcoidosis, 2 PS y 1 EMTC. FR: 1 Idiopático titulo 1:8. Anti DNA: 1 EMTC.

siendo las más frecuentes en nuestra población, por lo que recomendamos que el estudio inicial incluya, un hemograma, VHS, títulos ASO, PPD, radiografía de tórax, y de estimarse pertinente serologías para Mycoplasma pneumoniae, Bartonella henselae, etc. Dado que las enfermedades del sistema inmune aparecen segundas en frecuencia, nos parece razonable solicitar serología autoinmune en los casos en que la clínica lo sugiera y no haya sospecha de causa infecciosa. El estudio histológico sigue siendo necesario sólo en casos de presentación clínica atípica.

Las limitaciones de nuestro estudio son el carácter retrospectivo que pudo haber incidido en pérdida de información relevante como datos de recaída, uso de drogas (lo cual hubiese disminuido el porcentaje de casos idiopáticos) y duración de la enfermedad, y que las conclusiones obtenidas sólo pueden extrapolarse al ambiente hospitalario. Por otro lado, no todos los casos de EN se diagnosticaron con biopsia (Lo cual podría haber llevado a imprecisiones diagnósticas). Sin embargo, todos nuestros pacientes fueron evaluados por dermatólogos con vasta experiencia clínica, y siendo la placa eritematosa dolorosa tan característica, es poco probable el error diagnóstico en ausencia de diagnóstico cutáneo alternativo.

\section{Conclusión}

El eritema nodoso es un cuadro frecuente que plantea un amplio diagnóstico diferencial para el internista. Las diferencias en cuanto a la etiología 
del EN con otras series reportadas se dan fundamentalmente porque nuestra cohorte se generó en el escenario hospitalario. Es así como causas frecuentes de eritema nodoso, como son el uso de anticonceptivos orales y el embarazo, casi no se dieron en nuestra serie. En nuestro escenario hospitalario, aproximadamente un tercio de los casos fueron idiopáticos, y los casos secundarios se asociaron en su mayoría a cuadros infecciosos, siendo la sarcoidosis el cuadro no infeccioso más frecuentemente involucrado. La presencia de VHS elevada y leucocitosis tiende a hacer pensar más en cuadros secundarios que en idiopáticos. Una aproximación inicial razonable en pacientes hospitalizados por EN sería realizar una historia clínica y examen físico acucioso, un hemograma, VHS, títulos ASO, PPD, una imagen de tórax, y de estimarse pertinente según la clínica, solicitar otras serologías infecciosas e inmunológicas. No recomendamos realizar serologías de virus de hepatitis B, C ni VIH salvo que la sospecha clínica sea alta.

\section{Referencias}

1. Requena L, Sánchez E. Erythema Nodosum. Sem Cutan Med Surg 2007; 26: 114-25.

2. Requena L, Sánchez E. Erythema Nodosum. Dermatol Clin 2008; 26: 425-38.

3. Mana J, Marcoval J. Erythema nodosum. Clin Dermatol 2007; 25: 288-94.

4. García-Porrua C, González-Gay M, Vásquez-Caruncho M, López-Lázaro L, Lueiro M, et al. Erythema Nodosum: etiologic and predictive factors in a defined population. Arthritis Rheum 2000; 43: 584-92.
5. Mert A, Kumbasar H, Ozaras R, Erten S, Tasli L, et al. Erythema nodosum: an evaluation of 100 cases. Clin Exp Rheumatol 2007; 25: 563-70.

6. Cribier B, Caille A, Heid E, Grosshans E. Erythema nodosum and associated diseases. A study of 129 cases. Int J Dermatol 1998; 37: 667-72.

7. Kakourou T, Drosatou P, Psychou F, Aroni K, Nicolaidou P. Erythema Nodosum in children: a prospective study. J Am Acad Dermatol 2001; 44: 17-21.

8. Schwartz RA, Nervi SJ. Erythema Nodosum: A Sign of Systemic Disease. Am Fam Physician 2007; 75: 695-700.

9. Papagrigokari A, Gisondi P, Rosina P, Cannone M, Girolomoni G. Erythema Nodosum: etiological factors and relapses in a retrospective cohort study. Eur J Dermatol 2010; 20: 773-7.

10. Amal A, Mamdouh N, Ezz Sahar. Antistreptolysin O titer in health and disease: levels and significance. Pediatr Rep 2012; 4 (1): e8.

11. International Study Group for Behcet's Disease. Criteria for diagnosis of Behcet's disease. Lancet 1990; 335: 107880.

12. Uçkaya I, Ferry T, Stern R, Ambrosioni J, Gamulin A, Andrey A, et al. Use if serum antistreptolysin $\mathrm{O}$ titers in the microbial diagnosis of orthopedic infections. IJID July 2009, Volume 13, Issue 4, Pages 421-4.

13. Torinuki W, Funyu T. Adult Still's disease manifesting as erythema nodosum. Med Clin (Barc). 1991; 97 (19): 757.

14. Manresa JM, Caballol R, Grau A, Piñas I. Association of mixed connective tissue disease and erythema nodosum. Med Clin (Barc) 1991; 97 (19): 757.

15. Grunewald RW, Fiedler GM, Stöckmann F, Schauer A, Müller GA. Unusual diagnosis in recurrent arthritis, erythema nodosum and arrhythmia. Dtsch Med Wochenschr. 1997; 122 (49): 1516-20. 